\title{
Estimations of the critical temperatures for development of the pistachio psylla, Agonoscena pistaciae (Hemiptera: Psyllidae)
}

\author{
Mohammad Reza HASSANI ${ }^{1}$, Abbas ARBAB ${ }^{2}$, Hamzeh IZADI ${ }^{3}$ and Gadir NOURI-GANBALANI ${ }^{4}$ \\ ${ }^{1}$ Department of Plant Protection, Islamic Azad University, Rafsanjan Branch, Rafsanjan, Iran; e-mail: mreza.hassani@yahoo.com \\ ${ }^{2}$ Department of Plant Protection, Islamic Azad University, Takestan Branch, Takestan, Iran; e-mail: a.arbab@tiau.ac.ir \\ ${ }^{3}$ Vali-e-Asr University, Rafsanjan, Iran; e-mail: izadi@vru.ac.ir \\ ${ }^{4}$ University of Mohaghegh Ardabili, Ardabil, Iran; e-mail: gadirnouri@yahoo.com
}

Key words. Psyllidae, Agonoscena pistaciae, critical temperature, developmental rate

\begin{abstract}
The pistachio psylla, Agonoscena pistaciae Burckhardt \& Lauterer (Hemiptera: Psyllidae), is a major pest of pistachio trees throughout the pistachio producing regions in Iran. The effect of temperature on the developmental rates of eggs and nymphs of A. pistaciae was determined at different constant temperatures, i.e. $15,20,25,30,32.5$ and $35 \pm 0.5^{\circ} \mathrm{C}$. The relationships between temperature and developmental rates were described by linear and the non-linear Lactin models. These models were evaluated based on $R^{2}, R S S, A I C$ and $R_{\text {adj. }}^{2}$ The estimated value of the lower temperature threshold for egg, nymph and egg to adult development based on the linear model was $8.06,10.38$ and $9.97^{\circ} \mathrm{C}$, respectively, and based on the Lactin model was $8,11.55$ and $11.2^{\circ} \mathrm{C}$, respectively. Thermal constants estimated using the linear model, were 88.5, 243.90 and 333.33 DD, respectively, for egg, nymph and egg to adult development. These results indicate that the linear model gives a better description of the relationship between developmental rate and temperature for $A$. pistaciae than the non-linear model. These results could be incorporated into forecasting models used in the integrated pest management of this pest.
\end{abstract}

\section{INTRODUCTION}

Pistachio, Pistacia vera L., is one of the most important horticultural products in Iran. The pistachio psyllid, Agonoscena pistaciae Burckhardt \& Lauterer (Hemiptera: Psyllidae), is a major pest of pistachio trees in all the areas of Iran where this plant is grown. This pest sucks sap from the leaves, which damages the plant and reduces yield. This pest has several generations per year and is controlled by applying chemical insecticides several times each year (Razavi, 2005; Samih et al., 2005; Hassani, 2009). Temperature is an abiotic factor influencing the dynamics, rate of development, reproduction, mortality and survival of arthropod pests and their natural enemies (Campbell et al., 1974; Honek et al., 2003; Tokuda \& Matsumura, 2005; Ozder \& Saglam, 2008). The relationship between developmental rate and temperature is an important ecological variable in models of the population dynamics of insects (Wagner et al., 1984). The relationship between temperature and developmental rate could provide useful parameters for predicting the development of this pest and for determining the optimal time to release natural enemies (Arbab et al., 2008). It is easy to calculate the minimum temperature threshold using linear regression but not the maximum and optimum thresholds. Briere \& Pracros (1998) state that in insects, the relationship between developmental rate and temperature is nonlinear and asymmetrical. The linear model not only fits the linear portion of the relationship between developmental rate and temperature but provides an easy way of predicting the lower temperature threshold. Moreover, it is the only equation that can be used to calculate the thermal constant (Jervis \& Copland, 1996; Haghani et al., 2007). The lack of linearity in the developmental rate at low and high temperatures indicates that this model is unlikely to provide accurate predictions of the temperature thresholds. This has led to the development of several non-linear phenological models for use in integrated pest management programs (Wagner et al., 1984; Worner, 1992). The basic idea of non-linear regression is that a response $\mathrm{Y}$ is not linearly related to a predictor variable $\mathrm{X}$. In non-linear regression the prediction depends on one or more unknown parameters. Whereas linear regression is often used in empirical models, non-linear regressions are used when there are reasons for believing that the relationships between the response and the predictor has a particular functional form (Smyth, 2002).

The objective of this study was to determine whether a linear or the non-linear Lactin model better describes the effect of different constant temperatures on the developmental rate of the immature stages of $A$. pistaciae reared under laboratory conditions and in addition can be used to obtain estimates of the lower and upper temperature thresholds and optimum temperature. This information can be used to forecast the abundance and phenological development of this pest.

\section{MATERIAL AND METHODS}

\section{Rearing method and experimental conditions}

The eggs and nymphs of $A$. pistaciae were reared in the laboratory on pistachio leaf disks in plastic Petri-dishes $(6 \mathrm{~cm}$ diameter and $2 \mathrm{~cm}$ high). To provide ventilation, there was a hole in the middle of the lids covered with a piece of fine netting. Agar medium $\left(10^{-1} \mathrm{~g} \mathrm{~L}\right)$ was used as a source of moisture 
for the leaf disks. The disks of pistachio leaf (cultivar Ohadi) were cut to the same diameter as the dishes and each placed on a $3 \mathrm{~mm}$ thick layer of agar medium. To determine the effect of temperature on the development of $A$. pistaciae the eggs and nymphs were reared at a constant temperature of $15,20,25,30$, 32.5 or $35 \pm 0.5^{\circ} \mathrm{C}$, a photoperiod of $14 \mathrm{~L}: 10 \mathrm{D}$ and $50 \pm 5 \% \mathrm{RH}$ in a growth chamber. Eggs were obtained by releasing 5 mated females of $A$. pistaciae onto a pistachio leaf disk and removing them after $8 \mathrm{~h}$. To determine the development time of the eggs, all pistachio leaf disks were examined every $8 \mathrm{~h}$ and the number that had hatched recorded and the first-instar nymphs transferred to a new pistachio leaf disk. The nymphs were also examined at $8 \mathrm{~h}$ intervals to determine their developmental time. The nymphs were provided with fresh pistachio leaf disks every 3 days until they reached adulthood.

\section{Effect of temperature on development}

The results of rearing A. pistaciae at six constant temperatures were used to calculate the developmental times of the eggs and nymphs. Mean developmental rate of the different stages of $A$. pistaciae at the various temperatures was estimated using the following equation

$$
r(T)=\frac{1}{e\left[\sum \ln \frac{d t}{n}\right]}
$$

where $r(T)$ is the mean developmental rate at temperature $T$ $\left({ }^{\circ} \mathrm{C}\right) ; d t$, is the observed development time in days; and $n$ is number of observations. This method is recommended by Logan et al. (1976) and takes into account that the transformation of development time to developmental rate linearizes the relationship between $d t$ and $T\left({ }^{\circ} \mathrm{C}\right)$. These rates were used in the nonlinear regression model to calculate the critical temperatures $\left(t_{\min }, t_{\max }\right.$ and $\left.t_{\mathrm{opt}}\right)$.

\section{Linear model}

The linear model, Campbell et al. (1974), was used to estimate the lower temperature threshold $\left(T_{0}\right)$ and the thermal constant $(K)$ for the immature stages and egg to adult stage of $A$. pistaciae. The model of Campbell et al. (1974) is based on the linear regression equation, $r(T)=a+b T$, where $r(T)$ is the rate of development and $T$ is the temperature $\left({ }^{\circ} \mathrm{C}\right)$. The parameter $a$ is the intercept and $b$ the slope of the straight line. The lower temperature threshold is calculated as $T_{0}=-a / b$ and the thermal constant as $K=1 / b$.

The linear model only includes the rates of insect development $[r(T)]$ that are on the linear part of the developmental curve of an insect; for this reason, data values for egg at $35^{\circ} \mathrm{C}$ and for nymph and egg to adult at 32.5 and $35^{\circ} \mathrm{C}$ that deviated from the straight line were not included when this model was used to calculate the lower temperature threshold and thermal constant. The linear relation between temperature and developmental rate simplifies the mathematics associated with calculating durations, and is the basis for estimating the cardinal values ( $T_{0}$ and $K$ ) (Trudgill et al., 2005).

\section{Non-linear model}

A non-linear model (Lactin et al., 1995) was used to estimate the upper and lower temperature thresholds and the optimum temperature for the development of eggs, nymphs and egg to adult stage of $A$. pistaciae. Lactin et al. (1995) made two modifications to the Logan model (Logan et al., 1976), resulting in the following equation

$$
r(T)=e^{\rho . T}-e\left[\rho \cdot T_{\max }-\left(\frac{T_{\max }-T}{\Delta T}\right)\right]+\lambda
$$

where $T$ is the temperature $\left({ }^{\circ} \mathrm{C}\right.$ ) and $T_{\max }$ (upper temperature threshold), $\rho, \Delta T$ and $\lambda$ are fitted parameters. The parameters of the non-linear Lactin model were estimated using the non-linear regression model of Marquardt (1963) and JMP and SPSS (v. 9.0; SPSS 1999) statistical programs. The lower and upper temperature thresholds were estimated using these equations. The optimum temperature for development is that at which the rate of development curve reaches its maximum value (Arbab et al., 2008).

\section{Statistical analysis}

The normality and homogeneity of data were analyzed using one way ANOVA and the differences between means were determined using the least significant difference test with the $P$-value set at 0.05 .

\section{Evaluation of models}

Four criteria were used to assess the performance of the mathematical models:

1. The coefficient of determination $\left(R^{2}\right)$. High values of $R^{2}$ indicate a better fit.

2 . The residual sum of square (RSS). Low values of $R S S$ indicate a better fit.

The coefficient of determination and residual sum of square are commonly used for model evaluation.

However, the $R^{2}$ value is not appropriate for discriminating between models with different numbers of parameters because models with more parameters always provide a better fit. Therefore, two other statistics that are parameter independent were used.

3. The Akaike information criterion (AIC). With this criterion, the model with the lowest $A I C$ was sought, which is the function that minimizes the loss of information (Akaike, 1974; Ranjbar Aghdam et al., 2009). The $A I C$ was calculated using the following equation

$$
A I C=n \ln \left(\frac{S S E}{n}\right)+2 p
$$

where $n$ is the number of observations, $p$ is the number of model parameters including the intercept, and SSE is the sum of the squared error.

4. The adjusted coefficient of determination $\left(R_{\text {adj }}^{2}\right)$.

As $A I C$ is parameter independent a high value of $R_{\text {adj }}^{2}$ indicates a better fit (Rezaei \& Soltani, 1998). $R_{\text {adj }}^{2}$ was calculated using the following equation

$$
R_{a d j}^{2}=1-\left(\frac{n-1}{n-p}\right)\left(1-R^{2}\right)
$$

where $n$ is the number of observations, $p$ is the number of model parameters and $R^{2}$ is the coefficient of determination.

\section{RESULTS}

\section{Developmental time}

The developmental times of the eggs and nymphs of $A$. pistaciae at six constant temperatures on pistachio leaf disks are presented in Table 1 . The developmental time for eggs decreased from 15 to $32.5^{\circ} \mathrm{C}$ and for nymphs from 15 to $30^{\circ} \mathrm{C}$ and then increased from 30 to $35^{\circ} \mathrm{C}$. The developmental time from egg to adult obtained by summing the values for eggs and nymphs, i.e., the total devel-

TABLE 1. Developmental time (days) of different stages of $A$. pistaciae recorded at different constant temperatures.

\begin{tabular}{lcccccc}
\hline \multirow{2}{*}{ Stage } & \multicolumn{6}{c}{ Temperature $\left({ }^{\circ} \mathrm{C}\right)$} \\
\cline { 2 - 7 } & 15 & 20 & 25 & 30 & 32.5 & 35 \\
\hline Egg & 13.29 & 7.484 & 4.936 & 4.122 & 3.642 & 3.974 \\
Nymph & 45.17 & 30.10 & 15.33 & 12.38 & 20.254 & 22.48 \\
Egg to adult & 58.46 & 37.59 & 20.26 & 16.51 & 23.896 & 26.45 \\
\hline
\end{tabular}




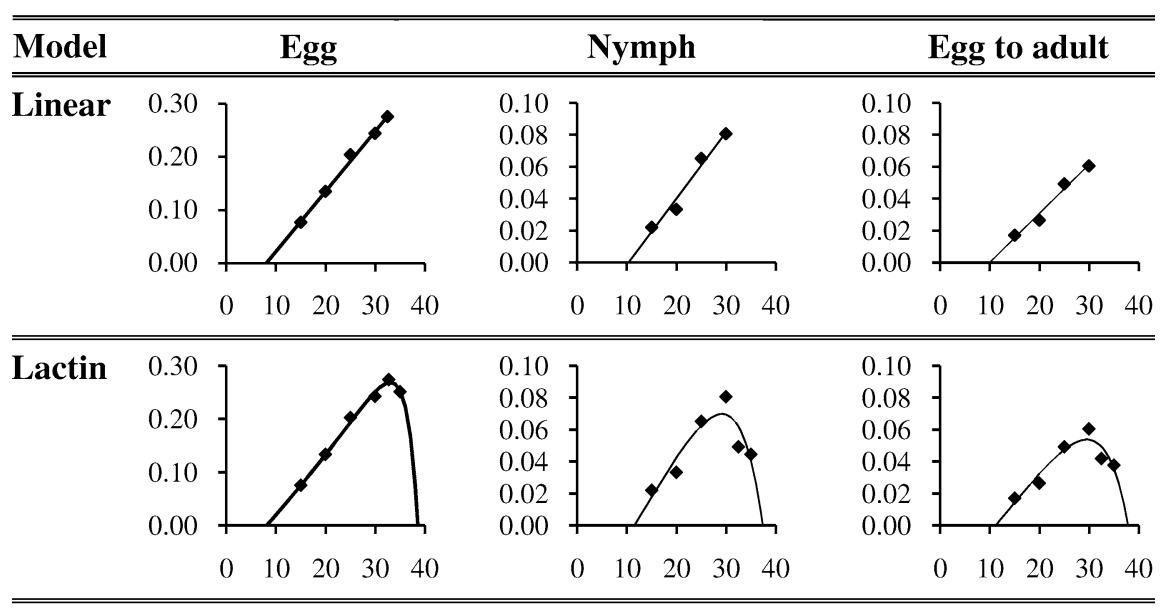

Fig. 1. The fit of a linear and the non-linear Lactin model to the relationships between the developmental rates $\left(\right.$ Day $\left.^{-1}\right)$ of eggs, nymphs and egg to adult stages of $A$. pistaciae recorded over a range of constant temperatures $\left({ }^{\circ} \mathrm{C}\right)$.

opmental time for $A$. pistaciae, ranged from 58.46 days at $15^{\circ} \mathrm{C}$ to 16.51 days at $30^{\circ} \mathrm{C}$.

The linear regression equations that describes the relationship between egg, nymph and egg to adult developmental rates and temperature is presented in Table 3 . The developmental time for egg at $35^{\circ} \mathrm{C}$ and and for nymph and egg to adult at 32.5 and $35^{\circ} \mathrm{C}$ were longer than predicted by the linear relationship between developmental rate and temperature. Thus, data for these temperatures were not included when the linear regression equation was used to obtain the lower temperature threshold and thermal constant.

The developmental rate of egg, nymph and egg to adult of $A$. pistaciae was used to evaluate whether the linear or the non-linear Lactin model better describes the relationship between developmental rate and temperature. All fitted parameters were estimated using linear and nonlinear regression analyses. The estimated values of the coefficients and parameters of the linear and non-linear models are presented in Table 3 .

\section{Linear model}

The linear regression equation describing the relationship between the developmental rates and temperature for egg, nymph and egg to adult stages of $A$. pistaciae are

TABLE 2. Values of the parameters of the linear and nonlinear Lactin models describing the effect of temperature on the developmental time of different stages of A. pistaciae.

\begin{tabular}{ccccc}
\hline Model & Parameters & Egg & Nymph & Egg to adult \\
\hline \multirow{4}{*}{ Linear } & $a$ & -0.0911 & -0.0432 & -0.0305 \\
& $b$ & 0.0113 & 0.0041 & 0.0030 \\
& $t_{\min }$ & 8.0619 & 10.3846 & 9.9673 \\
& $k$ & 88.4956 & 243.9024 & 333.3333 \\
\hline \multirow{6}{*}{ Lactin } & $\rho$ & 0.0098 & 0.00508 & 0.0037 \\
& $T_{\mathrm{L}}$ & 41.5776 & 48.4536 & 49.8132 \\
& $\Delta T$ & 2.1939 & 5.1621 & 4.9970 \\
& $\lambda$ & -1.0818 & -1.0595 & -1.0423 \\
& $t_{\min }$ & 8 & 11.55 & 11.2 \\
& $t_{\mathrm{opt}}$ & 32.75 & 29.2 & 29.6 \\
& $t_{\max }$ & 38.54 & 37.4 & 37.86
\end{tabular}

presented in Table 3 . The high values of the coefficients $R^{2}, R S S, A I C$ and $R_{\text {adj }}^{2}$ for egg, nymph and egg to adult stages of $A$. pistaciae indicate that the linear model adequately describes the relationship between developmental rate and temperature. The lower temperature threshold estimated by the linear model for egg, nymph and egg to adult stages was $8.06,10.38$ and $9.97^{\circ} \mathrm{C}$, respectively. The thermal constant for the egg, nymph and egg to adult stages was $88.5,243.90$ and $333.33 \mathrm{DD}$, respectively (Table 3).

\section{Non-linear model}

The non-linear Lactin model was used to describe the relationship between the developmental rate of $A$. pistaciae and temperature. The value of $R^{2}, R S S, A I C$ and $R^{2}$ adj and the parameters of this model are presented in Table 3. For the egg stage the Lactin model satisfactorily describes the relationship between the developmental rate and temperature. The $R^{2}$ coefficient for the Lactin model is 0.9584 (Table 2) and the RSS value is low (Table 2). Plots of the values for $r(T)$ and curves of the relationships between $r(T)$ and temperature for egg, nymph and egg to adult stages of $A$. pistaciae fitted using linear and nonlinear models are presented in Fig. 1.

TABLE 3. The coefficients and parameters of the linear and non-linear Lactin models fitted to the relationships between the rates of development at different temperatures of different developmental stages of $A$. pistaciae.

\begin{tabular}{cccc}
\hline \multirow{4}{*}{ Model } & Egg & Nymph & Egg to adult \\
\cline { 2 - 4 } & $R^{2}$ & $R^{2}$ & $R^{2}$ \\
& $R S S\left(\times 10^{-4}\right)$ & $R S S\left(\times 10^{-4}\right)$ & $R S S\left(\times 10^{-4}\right)$ \\
& $R^{2}{ }_{\text {adj }}$ & $R^{2}$ adj & $R^{2}$ adj \\
\hline \multirow{5}{*}{ Linear } & $A I C$ & $A I C$ & $A I C$ \\
& 0.9937 & 0.9664 & 0.9739 \\
& 1.6712 & 0.943 & 0.314 \\
& 0.9895 & 0.944 & 0.9565 \\
Lactin & -58.9813 & -62.3647 & -68.9627 \\
& 0.9584 & 0.8181 & 0.8734 \\
& 1.8259 & 4.1148 & 1.5388 \\
& 0.8960 & 0.5452 & 0.6835 \\
& -54.4001 & -49.5250 & -55.4265 \\
\hline
\end{tabular}


The values of the lower temperature threshold for egg, nymph and egg to adult predicted by the Lactin model are $8,11.55$ and $11.2^{\circ} \mathrm{C}$, respectively (Table 3 ).

The optimum temperature for the development of the eggs of $A$. pistaciae predicted by the Lactin model is 32.75 , which is $3^{\circ} \mathrm{C}$ higher than the values predicted for the nymph and egg to adult stages, 29.2 and $29.6^{\circ} \mathrm{C}$, respectively (Table 3 ). The upper temperature thresholds for the development of the egg, nymph and egg to adult stages are $38.54,37.4$ and $37.86^{\circ} \mathrm{C}$, respectively.

The value of $\lambda$ estimated using the model of Lactin et al. (1995) was less than zero (Table 3), which indicates that this model can be used to calculate the lower temperature threshold. The lower temperature threshold for eggs determined by the Lactin model is $8^{\circ} \mathrm{C}$ (Table 3 ). The low value of $R^{2}$ and high values of RSS and $A I C$ for the nymph and egg to adult stages of $A$. pistaciae indicate that the non-linear Lactin model does not describe the results as well as the linear model. The high value of $R^{2}$ and low values of $R S S$ and $A I C$ for the nymph and egg to adult stages of $A$. pistaciae indicate that the fit obtained using the linear model more accurately describes the relationship between developmental rate and temperature. The linear model is recommended for describing the relationship between developmental rate and temperature for the nymph and egg to adult stages of $A$. pistaciae.

\section{DISCUSSION}

Temperature is an important factor influencing the dynamics of arthropod pests and their natural enemies (Pedigo, 2002; Gullan \& Cranston, 2005). Knowledge of the temperature thresholds of pests has practical implications as they can be incorporated in phenological models. Phenological models based on temperature have been developed to forecast biological events of insects (Diaz et al., 2007). The shortest developmental time for the eggs of $A$. pistaciae was recorded at $32.5^{\circ} \mathrm{C}$ and it increased from 32.5 to $15^{\circ} \mathrm{C}$. The shortest developmental time for the nymphs was recorded at $30^{\circ} \mathrm{C}$, below which it increased as temperature decreased and at $35^{\circ} \mathrm{C}$ it was longer than at $30^{\circ} \mathrm{C}$.

The lower temperature threshold for egg development predicted by the Lactin model is $8^{\circ} \mathrm{C}$, which is similar to the value predicted by the linear model $\left(8.06^{\circ} \mathrm{C}\right)$. The Lactin model successfully describes the effect of temperature on the development of many arthropods (Kontodimas et al., 2004). The parameter $\lambda$ allows the curve to intersect the abscissa at sub-lethal temperatures and thus the prediction of the lower temperature threshold (Lactin et al., 1995). For nymphs and the egg to adult stage the fit of the linear model had a higher $R^{2}$ and lower $R S S$ and $A I C$ values than that of the Lactin model and therefore the linear model provides the best description of the results (Table 2). Based on the linear model the lower temperature threshold for nymph and egg to adult stages are 10.38 and $9.97^{\circ} \mathrm{C}$, respectively. The non-linear Lactin model provided a poorer description of the results. For eggs the linear model also provided a better description of the results than the non-linear Lactin model. Trudgill et al. (2005) suggest that the linear thermal time model applies to both invertebrates and plants and is conceptually and practically useful.

The lower temperature threshold for the egg to adult stage is $9.97^{\circ} \mathrm{C}$, which is $1^{\circ} \mathrm{C}$ lower than that recorded for the male and female of 10.90 and $10.71{ }^{\circ} \mathrm{C}$, respectively, in a previous study (Mehrnejad, 1998). The thermal constant for the development from egg to adult is 333.33 DD. The values recorded for male and female $A$. pistaciae in a previous study are 227.27 and $238.10 \mathrm{DD}$, respectively (Mehrnejad, 1998). The thermal constant determined in this study is about 100 DD higher than that recorded by Mehrnejad (1998). The thermal constants for A pistaciae eggs (88.5 DD), nymphs (243.90 DD) and egg to adult (333.33 DD) are close to those reported for Psylla pyricolla egg (84 DD), nymph (252 DD) and egg to adult stages (336 DD) (Brunner, 1984). The lower temperature threshold for Heteropsylla cubana Crawford recorded in the laboratory and the field are $8.6^{\circ} \mathrm{C}$ and $9.6^{\circ} \mathrm{C}$, respectively (Geiger \& Gutierrez, 2000). These results are close to those recorded for $A$. pistaciae. The lower temperature threshold for A. pistaciae determined in this study is lower than that of most coccinellid predators of this pest, such as Oenopia conglobata contaminata Menetries (12.76), Coccinella undecimpunctata aegyptiaca Reiche (14.02) and Hippodamia variegata (Goeze) (14.41) (Jalali, 2001; Mehrnejad \& Jalali, 2004). These results indicate that these predators will appear in pistachio orchards after $A$. pistaciae and disappear before this pest. The thermal constant is used to monitor and model insect development for predictive and descriptive purposes (Worner, 1988). The relationship between insect developmental rate and temperature reported here is an important ecological variable, which should be included in models of the population dynamics of this pest. Forecasting models can be used to predict when to start monitoring a pest in the field or the optimum time to apply control measures, such as insecticides or release of natural enemies (Akers \& Nielsen, 1984; Brunner, 1984; Graf et al., 2006; Diaz et al., 2007; Arbab et al., 2008). Such a forecasting model could be incorporated into integrated pest management programs of this pest and used to predict when best to either apply an insecticide or release natural enemies.

ACKNOWLEDGEMENTS. The authors thank M. Basirat for technical advice. We thank two anonymous referees for valuable comments on the manuscript. This study was supported by the Islamic Azad University, Rafsanjan Branch, Rafsanjan, Iran.

\section{REFERENCES}

AKaIKe H. 1974: A new look at the statistical model identification. IEEE Trans. Automat. Contr. 19: 716-723.

Akers R.C. \& Nielsen D.G. 1984: Predicting Agrillus anxius (Col.: Buprestidae) adult emergence by heat unit accumulation. J. Econ. Entomol. 77: 1459-1463.

Arbab A., Kontodimas D.C. \& Mcneill M.R. 2008: Modeling emberyo develioment of Sitona discoideus Gyllenhal (Coleoptera: Curculionidae) under constant temperature. Environ. Entomol. 37: 1381-1388. 
Briere J.F. \& Pracros P. 1998: Comparison of temperaturedependent growth models with the development of Lobesia botrana (Lepidoptera: Tortricidae). Environ. Entomol. 27: 94-101.

BRUNNER J.F. 1984: The development, distribution and sampling for the pear psyllid, Psylla pyricola. IOBC/WPRS Bull. 7: $81-96$.

Campbell A., Frazer B.D., Gilbert N., Gutierrez A.P. \& MACKAUER M. 1974: Temperature requirements of some aphids and their parasites. J. Appl. Ecol. 11: 431-438.

Diaz B.M., Muniz M., Barrios L. \& Fereres A. 2007: Temperature thresholds and thermal requirements for development of Nasonovia ribisnigri (Hem.: Aphididae). Environ. Entomol. 36: $681-688$.

Geiger C.A. \& Gutierrez A.P. 2000: Ecology of Heteropsylla cubana (Hom.: Psyllidae): psyllid damage, tree phenology, thermal relations, and parasitism in the field. Environ. Entomol. 29: 76-86.

Graf B., Hohn H.U., Hohn H. \& Samietz J. 2006: Temperature effects on egg development of the rosy apple aphid and forecasting of egg hatch. Entomol. Exp. Appl. 119: 207-211.

Gullan P.J. \& Cranston P.S. 2005: The Insects: An Outline of Entomology. 3rd ed. Blackwell, Oxford, 505 pp.

Haghani M., Fathipour Y., Talebi A.A. \& Baniameri V. 2007 Temperature-dependent development of Diglyphus isaea (Diptera: Agromyzidae) on cucumber. J. Pest Sci. 80: 71-77.

HaSSANI M.R. 2009: Bioecology and Economic Injury Level of Agonoscena pistaciae (Hem.: Psyllidae) in Rafsanjan Region of Iran. Ph.D. Thesis, Islamic Azad University, Tehran, 105 pp.

Honek A., Jarosik V. \& Martinkova Z. 2003: Effect of temperature on development and reproduction in Gastrophysa viridula (Coleoptera: Chrysomelidae). Eur. J. Entomol. 100: 295-300.

Jalali M.A. 2001: Study of Food Consumption in Predatory Beetles (Col.: Coccinellidae) of the Common Pistachio Psyllid, Agonoscena pistaciae (Hem.: Psylloidea) in Rafsanjan and Compiling a Life Table in the Controlled Condition. M.Sc. Thesis, Shiraz University, Shiraz, 120 pp.

Jervis M.A. \& Copland M.J.W. 1996: The life cycle. In Jervis M. \& Kidd N. (eds): Insect Natural Enemies, Practical Approaches to their Study and Evaluation. Chapman Hall, London, pp. 63-161.

Kontodimas D.C., Eliopoulos P.A., Stathas G.J. \& Economou L.P. 2004: Comparative temperature-dependent development of Nephus includens (Kirsch) and Nephus bisignatus (Boheman) (Coleoptera: Coccinellidae), preying on Planococcus citri (Risso) (Homoptera: Pseudococcidae): evaluation of a linear and various non-linear models using specific criteria. Environ. Entomol. 33: 1-11.

Lactin D.J., Holliday N.J., Johnson D.L. \& Craigen R. 1995: Improved rate model of temperature-dependent development by arthropods. Environ. Entomol. 24: 68-75.

Logan J.A., Wollkind D.J., Hoyt S.C. \& Tanigoshi L.K. 1976: An analytic model for description of temperature dependent rate phenomena in arthropods. Environ. Entomol. 5: $1133-1140$.

MARQUARDT D.V. 1963: An algorithm for least squares estimation of nonlinear parameters. J. Soc. Ind. Appl. Math. 11: 431-441.

Mehrnejad M.R. 1998: Evaluation of the Parasitoid Psyllaephagus pistaciae (Hym.: Encyrtidae) as a Biocontrol Agent of the Common Pistachio Psylla Agonocena pistaciae (Hem.: Psylloidea). Ph.D. Thesis, University of London, $271 \mathrm{pp}$.

MeHRNEJAD M.R. \& JALALI M.A. 2004: Life history parameters of the coccinellid beetle, Oenopia conglobata contaminata, an important predator of the common pistachio psylla, Agonoscena pistaciae (Hemiptera: Psyllidae). Biocontr. Sci. Technol. 14: 701-711.

OzdER N. \& SAGLAM O. 2008: Effect of temperature on the biology of Tuberolachnus salignus (Gmlin) Sternorrhyncha: Aphididae on Salix alba. J. Centr. Eur. Agric. 9: 171-176.

Pedigo L.P. 2002: Entomology and Pest Management. 4th ed. Prentice Hall, Upper Saddle River, NJ, 742 pp.

Ranjbar Aghdam H., Fathipour Y., Radjabi G. \& Rezapanah M. 2009: Temperature-dependent development and temperature thresholds of codling moth (Lepidoptera: Tortricidae) in Iran. Environ. Entomol. 38: 885-895.

RaZAVI S. 2005: Pistachio production, Iran vs. the world. In: $I V$ International Symposium on Pistachios and Almonds. ISHS Tehran, Iran, May 22-25. p. 209.

Rezaei A. \& Soltani A. 1998: An Introduction to Applied Regression Analysis. University of Technology, Isfahan, Iran.

Samih M.A., Alizadeh A. \& Saberi Riseh R. 2005: Pistachio Pests and Diseases in Iran and their IPM. Organization of Jihad-e-University, Tehran, $301 \mathrm{pp}$.

SмYтн G.K. 2002: Nonlinear regression. In El-Shaarawi A.H. \& Piegorsch W.W. (eds): Encyclopedia of Environmetrics. Vol. 3. A.H. Wiley, Chichester, pp. 1404-1411.

SPSS 1999: SPSS Base 9.0 User's Guide. SPSS, Chicago, IL.

ToKuda M. \& Matsumura M. 2005: Effect of temperature on the development and reproduction of the maize orange leafhopper Cicadulina bipunctata (Melichar) (Hom.: Cicadellidae). J. Appl. Entomol. Zool. 40: 213-220.

Trudgill D.L., Honek A., Li D. \& Van Straalen N.M. 2005: Thermal time-concepts and utility. Ann. Appl. Biol. 146: $1-14$.

Wagner T.L., Wu H.P., Sharpe J.H., Schoolfield R.H. \& Coulson R.N. 1984: Modeling insect development rates: a literature review and application of a biophysical model. Ann. Entomol. Soc. Am. 77: 208-225.

WORNER S.P. 1988: Evaluation of diurnal temperature models and thermal summation in New Zealand. J. Econ. Entomol. 81: 9-13.

WoRNer S.P. 1992: Performance of phenological models under variable temperature regimes: consequences of the Kaufmann or rate summation effect. Environ. Entomol. 21: 689-699.

Received June 8, 2010; revised and accepted March 11, 2011 